\title{
BMJ Open Does pain influence force steadiness? A protocol for a systematic review
}

\author{
Michail Arvanitidis (D) , ${ }^{1}$ Deborah Falla (D) , ${ }^{1}$ Andy Sanderson (D) , ${ }^{1,2}$ \\ Eduardo Martinez-Valdes (i) ${ }^{1}$
}

To cite: Arvanitidis M, Falla D, Sanderson A, et al. Does pain influence force steadiness? A protocol for a systematic review. BMJ Open 2021;11:e042525. doi:10.1136/ bmjopen-2020-042525

- Prepublication history for this paper is available online. To view these files, please visit the journal online (http://dx.doi org/10.1136/bmjopen-2020042525).

Received 07 July 2020 Revised 26 November 2020 Accepted 30 December 2020

Check for updates

(C) Author(s) (or their employer(s)) 2021. Re-use permitted under CC BY-NC. No commercial re-use. See rights and permissions. Published by BMJ.

${ }^{1}$ Centre of Precision Rehabilitation for Spinal Pain (CPR Spine), School of Sport, Exercise and Rehabilitation Sciences, College of Life and Environmental Sciences, University of Birmingham, Birmingham, UK

${ }^{2}$ Department of Sport and Exercise Sciences, Musculoskeletal Science and Sports Medicine Research Centre, Manchester Metropolitan University, Manchester, UK

Correspondence to Dr Eduardo Martinez-Valdes; E.A.MartinezValdes@bham. ac.uk

\begin{abstract}
Introduction Performing contractions with minimum force fluctuations is essential for everyday life as reduced force steadiness impacts on the precision of voluntary movements and functional ability. Several studies have investigated the effect of experimental or clinical musculoskeletal pain on force steadiness but with conflicting findings. The aim of this systematic review is to summarise the current literature to determine whether pain, whether it be clinical or experimental, influences force steadiness.

Methods and analysis This protocol for a systematic review was informed and reported in line with the Preferred Reporting Items for Systematic Reviews and Meta-Analyses Protocols and the Cochrane Handbook for Systematic Reviews of Interventions. Key databases will be searched from inception to 31 August 2020, including MEDLINE, EMBASE, PubMed, CINAHL Plus, ZETOC and Web of Science. Grey literature and key journals will be also reviewed. Risk of bias will be assessed with the Newcastle-0ttawa tool, and the quality of the cumulative evidence assessed with the Grading of Recommendations, Assessment, Development and Evaluation guidelines. If homogeneity exists between groups of studies, metaanalysis will be conducted. Otherwise, a narrative synthesis approach and a vote-counting method will be used, while the results will be presented as net increases or decreases of force steadiness.

Ethics and dissemination The findings will be presented at conferences and the review will be also submitted for publication in a refereed journal. No ethical approval was required.

PROSPERO registration number CRD42020196479
\end{abstract}

\section{INTRODUCTION}

During a voluntary movement of submaximal effort, the force produced by an individual is not constant, instead, it fluctuates around an average value. ${ }^{1}$ This variability of force is the consequence of neuromuscular noise and other environmental influences. ${ }^{2}$ The ability of an individual to produce a steady force during a submaximal voluntary contraction is defined as force steadiness (or torque steadiness) and is an important aspect of force control. ${ }^{3}$ Being able to perform contractions with minimum force fluctuations is essential for physical function and its impairment could influence the precision of voluntary

\section{Strengths and limitations of this study}

This will be the first systematic review to synthesise evidence and examine the effect of experimental and/or clinical pain on force steadiness.

- The effect of experimental or clinical pain on force steadiness will be considered for a wide range of joints and muscles during different contraction types of varying force.

- This protocol was written in accordance with the recommendations provided by the Cochrane Handbook for Systematic Reviews of Interventions.

movements. This could affect the joints' dynamic stability, coordination and potentially result in altered joint kinematics and overall function. ${ }^{4-8}$

Smooth force generation is highly dependent on the sense of force, which is part of proprioception. $^{9}$ Multiple neuromuscular receptors, including Golgi tendon organs, muscle spindles (proprioceptors) and pressure-sensitive skin receptors contribute to the perception of force, by detecting mechanical tissue changes and transmitting action potentials to the central nervous system (CNS). ${ }^{10}$ The proprioceptive information (afferent inflow) from these receptors enters the spinal cord dorsal horn and then ascends via the dorsal column medial lemniscus pathway to the cerebral cortex (conscious path) or via the spinocerebellar pathways to the cerebellum (unconscious path).$^{11}$ Processing of proprioceptive afferent information can occur at all levels of the CNS, while it is consciously appreciated in the primary somatosensory area of the cerebral cortex. ${ }^{11}$ In this area, proprioceptive information is integrated with other somatosensory, vestibular and visual information. ${ }^{11}$ Then, a descending cerebral (efferent) motor command is sent to the tissues involved, resulting in an appropriate action. ${ }^{9}$ For this interactive and complex process, continuous monitoring and modulation of ascending and descending information is required, based on 
the proprioceptive input, motor output, perception and the resulting action. ${ }^{9}$ Therefore, proprioception is of vital importance in force control and proprioceptive deficits could possibly severely impair an individual's ability to maintain a steady force. ${ }^{12}$ Assessment of force steadiness is one way to evaluate sense of force and it is usually quantified either in absolute terms as the standard deviation (SD) of the force (or torque) signal or in relative terms as the coefficient of variation $(\mathrm{CoV})$ over time. ${ }^{13-15}$ Higher values of both $\mathrm{SD}$ and $\mathrm{CoV}$ represent poorer control of muscle force. During this process, visual feedback of the force can either be provided to the individual or not. Indeed, studies have shown that force steadiness can be influenced by the visual system. ${ }^{16}$

It is well known that pain alters the way that we move and that the related changes can persist even after the resolution of pain. ${ }^{17} 18$ Additionally, abundant research has shown that in the presence of acute, chronic or experimentally evoked pain, proprioception is usually reduced, likely due to altered afferent input from the painful area and/or central factors. ${ }^{911}{ }^{19-22}$ It is believed that the afferent information received from these receptors could interfere with the nociceptive input at various neurophysiological levels of processing (ie, cortical, spinal or peripheral), as both travel through a common complex neurological pathway and suppression of the proprioceptive input by nociceptive signals could occur (in the periphery and/or in the somatosensory cortex and/ or during the descending proprioceptive command). ${ }^{9}$ Additionally, disruption of musculoskeletal tissues and concurrent damage or destruction of mechanoreceptors can occur in the presence of a musculoskeletal injury (trauma) which could further disrupt proprioception. ${ }^{11}$

Considering the above, various studies have evaluated the influence of clinical or experimental pain on force steadiness during submaximal isometric or dynamic tasks. Among those, some have shown that people with painful musculoskeletal conditions such as neck pain ${ }^{23}$ or patellofemoral pain syndrome ${ }^{24}$ present with reduced force steadiness compared with asymptomatic individuals during isometric neck flexion and knee extension tasks respectively. Others have shown that short-term experimental muscle pain decreases force steadiness of upper and lower limb muscles during isometric contractions. ${ }^{25-27}$ However, there are also other studies with contradicting results, showing no difference in force steadiness with experimentally induced pain within the anterior tibialis muscle during isometric ankle dorsi-flexion tasks ${ }^{28}$ and in clinical conditions such as chronic elbow pain during isometric wrist extensions ${ }^{29}$ and shoulder impingement during isometric shoulder external/internal rotations. ${ }^{30}$ Finally, others showed that force steadiness deficits in people with neck, shoulder pain or knee osteoarthritis were apparent only during specific movements and/or during the exertion of high forces. ${ }^{13} 3132$

To our knowledge, no previous report has systematically reviewed the available body of literature investigating the influence of pain on force steadiness. Thus, the aim of this systematic review is to summarise the current literature to determine whether pain, whether it be clinical or experimental, influences force steadiness.

\section{METHODS}

This systematic review protocol is reported according to the Cochrane Handbook for Systematic Reviews of Interventions, the Conducting Systematic Reviews and Metaanalyses of Observational Studies of Etiology (COSMOS-E) guidance and the Preferred Reporting Items for Systematic Reviews and Meta-Analyses (PRISMA) Protocol 2015 checklist (online supplemental file 1) ${ }^{33-36}$ The Cochrane Handbook mainly focuses on the synthesis of evidence from intervention studies which is not related to this systematic review, as such research question is commonly addressed by observational studies. However, we followed these guidelines in addition to the COSMOS-E guidance for the following reasons: First, there are many similarities in the general structure and procedures used in both types of reviews. Second, the information within the Cochrane Handbook is described in more detail, and contains some information related to observational studies and, third, to date, widely accepted standards of systematic reviewsmeta-analysis of observational studies are lacking. ${ }^{37} \mathrm{We}$ are aware though that methodological differences exist, and thus particular attention will be given to certain steps of the conducted process (eg, choice of statistical methods, sources of heterogeneity, etc) ${ }^{37}$

\section{Eligibility criteria}

The eligibility criteria for this systematic review were delineated according to the Population, Intervention, Comparison, Outcomes and Study design (table 1)..$^{35} 38$ However, due to the nature of the studies that will be included in this review, 'Intervention' was rephrased to 'Indicator' as done previously. ${ }^{39}$

\section{Information sources}

The following electronic databases will be searched from inception to 31 August 2020: MEDLINE (Ovid Interface), EMBASE (Ovid Interface), PubMed, CINAHL Plus (EBSCO Interface), ZETOC and Web of Science (Clarivate Analytics). Database-specific search strategies have been developed and they include medical subject headings (MeSH) where appropriate. MeSH terms were included to enhance our research strategy by making it more efficient and better informed. ${ }^{40}$

In addition to database searching, handsearching of key journals will be conducted too. This will include the following journals: Journal of Electromyography and Kinesiology, Clinical Biomechanics, Muscle \& Nerve, Journal of Orthopaedic \& Sports Physical Therapy, Journal of Science and Medicine in Sport, Isokinetics and Exercise Science and Journal of Applied Physiology. Additionally, any relevant in-progress work that has not been published yet, will be identified by contacting relevant authors in the field. To minimise the risk of publication 
Table 1 Inclusion and exclusion criteria

Population Studies will be included if the population of interest is (1) adults aged $\geq 18$ years old, with (2) musculoskeletal pain (clinical), that is, 'pain experienced in muscles, tendons, bones or joints that arises from an underlying disease classified elsewhere' - chronic secondary musculoskeletal pain $\left({ }^{78}\right)$ or 'pain that is characterised by significant emotional distress or functional disability, and cannot be attributed directly to a known disease or damage process' - chronic primary musculoskeletal pain $\left({ }^{7879}\right.$ and/or (3) experimentally induced musculoskeletal pain, that is, musculoskeletal pain experienced due to sensitisation of nociceptors located in subcutaneous tissue (eg, electrical stimulation of the muscle, chemical stimulation of the muscle and/or joint) $\left({ }^{80}\right)$ and $(4)$ a comparative control group of asymptomatic individuals is present or individuals are exposed to different condition themselves (ie, cross-over design, where participants are assessed when they do not experience pain, when they do and after the painful experience subsides). There will be no restrictions in terms of gender and/or ethnicity. Studies in which pain is evoked by exercise (delayed onset of muscle soreness) and/or muscle fatigue will be excluded. Studies consisting of individuals with musculoskeletal pain who have been diagnosed with an underlying medical pathology or disorder (eg, muscular dystrophies, neurological disorders, systemic inflammatory diseases) and/or previously had a surgery and/or fracture related to the area of interest, will be excluded. This is because the main outcome of this study is to check the effect of pain on force steadiness in clinical populations and we wanted to avoid the confounding effects of the surgery (ie, tissue damage, decrease in muscle mass) on steadiness.

\begin{tabular}{|c|c|}
\hline Indicator & $\begin{array}{l}\text { Eligible studies will be those which include the use of a dynamometer or any other device (eg, force or torque } \\
\text { sensors) to measure force or torque steadiness or any equivalent to those (eg, force or torque or moment } \\
\text { variability). However, any studies using force platforms to measure stance steadiness (eg, while standing) will be } \\
\text { excluded. Both studies that provided visual feedback of individual's force or torque output during (at least in a } \\
\text { part of) the trial or did not provide visual feedback at all, will be included. All types of contractions which were } \\
\text { measured at an absolute force or torque level or any level relative to individual's maximal voluntary contraction } \\
\text { (MVC) will be included without any restriction. There will be also no restriction for the side evaluated (ie, } \\
\text { dominant or non-dominant). }\end{array}$ \\
\hline Outcomes & $\begin{array}{l}\text { The outcome of interest is the measurement of force or torque steadiness. Any measure related to force or } \\
\text { torque steadiness will be included, including CoV (ie, the ratio of the SD of the force/torque signal to the mean } \\
\text { force/torque exerted during the force/torque-matching task, multiplied by } 100), \mathrm{SD} \text {, root mean square error. } \\
\text { ( }{ }^{158182} \text { ) Only quantitative studies that measure force/torque steadiness or variability will be included. Studies } \\
\text { measuring other aspects of muscle force control (eg, force accuracy) will be excluded. }\end{array}$ \\
\hline
\end{tabular}

CoV, coefficient of variation; PRISMA, Preferred Reporting Items for Systematic Reviews and Meta-Analyses; SD, standard deviation.

bias, grey literature will be included too, and it will be accessed via the British national bibliography for report literature, OpenGrey database, ProQuest Dissertations \& Theses Global and EThOs. The World Congress of Biomechanics, International Society of Biomechanics, International Society of Electrophysiology and Kinesiology and the World Confederation for Physical Therapy congresses will be assessed too, from 2016 to 2020. If deemed necessary, the authors of potentially eligible studies will be contacted to check/confirm whether they had published their study or not. Previous systematic reviews on the same topic will be searched too if applicable. However, to our knowledge no previous systematic review exists on this topic. Moreover, to further minimise the risk of publication bias the reference lists of the included studies will be handsearched for additional relevant studies that have been missed with the search. ${ }^{41}$

\section{Search strategy}

The search strategy will be conducted by the lead author (MA) with no restrictions in terms of the date, format, design, region or language. To ensure that the search will be comprehensive and reproducible, it was developed after completion of scoping searches and with help provided by an experienced Health Sciences Librarian, member of the Research Skills Team of the University of Birmingham. A complete electronic search strategy for the MEDLINE (Ovid Interface) database can be found in the online supplemental file 2. This search strategy uses a combination of MeSH (including exploded terms) 
and keyword searching, to ensure maximal retrieval. ${ }^{42}$ Appropriate adjustments will be performed to the main search strategy, to fully adapt it to the other databases. These adjustments will include changes in terms of MeSH and syntax. For instance, the ADJ operator will change to 'NEAR' for the Web of Science database and to ' $\mathrm{N}$ ' for the CINAHL Plus (EBSCO Interface). However, consistency will be ensured at all times.

\section{Data management}

One reviewer (MA) will import all search results into EndNote X9 (Clarivate Analytics) reference management software. This will include citations and abstracts of potentially eligible studies identified and it will allow the identification and removal of any duplicates, before the start of the screening process. Then, the full text of the remaining potential relevant studies will be retrieved and saved into the same software. The final list of the potentially eligible studies (abstracts and full texts) will be saved in separate folders; one for each independent reviewer (MA and AS). Screening forms that reflect the aforementioned inclusion/exclusion criteria will be developed, piloted and used for the screening process.

\section{Study selection}

The screening forms will be pilot tested first by both reviewers on a small number of articles, to ensure their effectiveness. The screening process will begin with the screening of the titles/abstracts of the identified studies against the inclusion/exclusion criteria by the two reviewers (MA and AS) independently. Using the screening forms, the reviewers will subcategorise the studies into definitely eligible, definitely ineligible or doubtful. ${ }^{41}$ Therefore, any obviously irrelevant studies and relevant studies will be excluded and included respectively. In the event of doubtful categorisation, the reviewer will read the full text for further clarification. If uncertainty still exists, a meeting will be arranged between the two reviewers to discuss and decide whether this study should be included or not. If the two reviewers do not reach to an agreement after discussion, when a study's eligibility is unclear or in any other event of disagreement, a third independent reviewer (EM-V) will mediate the process. If further information is required to support the screening process, the authors of the study will be contacted.

The two reviewers will then independently examine the full text of all remaining studies for compliance with the eligibility criteria. Again, the third reviewer will support the process if necessary. During both the title/abstract and full-text screening phases, agreement between the two reviewers will be assessed with the kappa ( $\kappa)$ statistic. ${ }^{43}$ According to Orwin ${ }^{44}$ fair agreement will be reflected in values of kappa statistic between 0.40 and 0.59 , good agreement in values between 0.60 and 0.74 and when the values are $\geq 0.75$, the agreement will be considered as excellent. Information regarding the excluded studies will be reported within the PRISMA flow diagram.

\section{Data collection process}

A data extraction form will be developed, based on the checklist for data collection provided by Li et al, ${ }^{4343}$ and it will be driven by the objective of this review and the inclusion criteria. Pilot testing of this form will be performed on a small number of randomly chosen studies prior to its use; to ensure its effectiveness in collecting all the necessary information. MA will then extract and collate all the information and the accuracy of this process will be checked by AS. If any discrepancies exist, discussion between the two reviewers will be the first step to resolve them, but if the two reviewers do not reach to an agreement, the third reviewer (EM-V) will review the discrepancy. Author and/or journal details will not be concealed from the two reviewers.

If a study consists of ambiguous or inconclusive results, or any crucial information (eg, unpublished data) that needs to be extracted is missing, the authors will be contacted, and they will be asked to reply within 2 weeks. If the corresponding authors do not respond within the requested time period and further clarification is vital to classify a study as eligible, this study will be excluded under the following reason: 'for ambiguity'. If multiple versions of the same study exist, they will be collated, and the primary authors will be contacted for further clarification. Similarly, if two or more potentially eligible studies appear to use the same sample, the authors will be contacted to clarify whether the results are duplicated or not. On both occasions, any duplicates will be removed, one version will be selected and justified.

\section{Data items}

Items to be extracted from the eligible studies are presented in table 2. If a study consists of additional groups outside of this reviews' population of interest (ie, other than an asymptomatic control group and clinical pain or experimental pain group or both), these studies will be considered eligible but only the data from the groups of interest will be extracted.

\section{Risk of bias}

Two reviewers (MA and AS) will independently appraise the included studies for risk of bias (ROB) using the Newcastle-Ottawa Scale (NOS) ${ }^{45}$ Assessing primary studies for susceptibility to bias constitutes an essential part of the systematic review conduction process, as inclusion of studies with high ROB may lead to invalid conclusions. ${ }^{464}$ A large number of tools to appraise bias in observational epidemiological studies exist, however, there is no consensus among the health assessment groups about which one is the most appropriate to use. ${ }^{48-52}$ Therefore, the choice of the suitable tool is not straightforward.

The NOS scale is the most commonly used tool to assess ROB in observational studies, even though the results concerning its reliability and validity are mixed, showing in some instances lower inter-rater reliability when compared with other tools. ${ }^{4752-55}$ Our decision to use this 
Table 2 Characteristics of included studies

Date of data extraction:

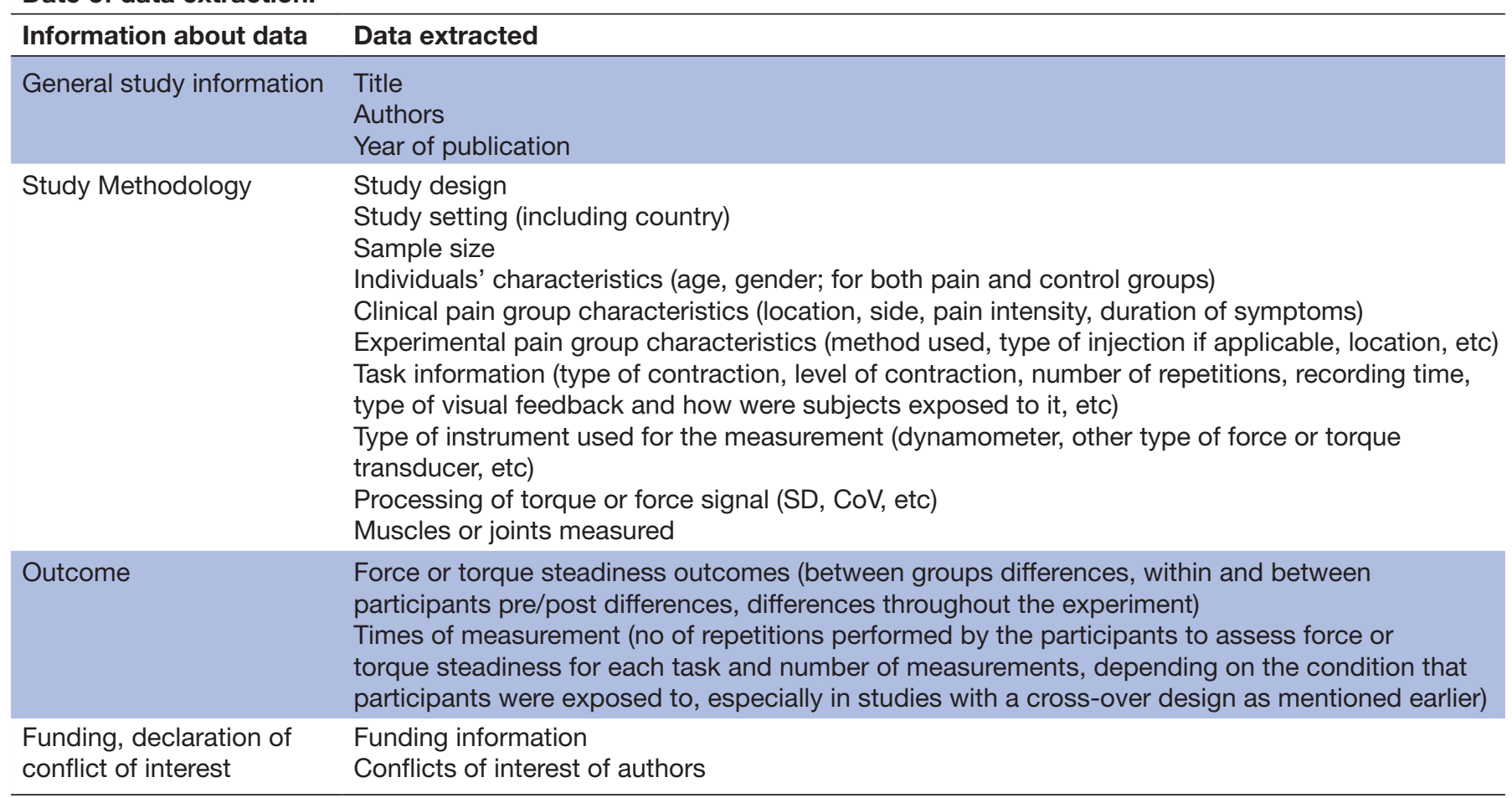

CoV, coefficient of variation; SD, standard deviation.

tool is supported by Hootman et $a \tilde{l}^{33}$ who have previously suggested that the NOS tool is still the preferable tool to assess ROB in observational studies because (1) it has good to moderate reliability, (2) it is easy to implement and (3) it has been argued to have some degree of face validity across observational designs. Losilla $e t a t^{47}$ have also more recently shown that the NOS tool has moderate to good inter-rater reliability, further supporting the findings of Hootman et $a \bar{p}^{3}$ and adding short scoring time as an additional strength. Additionally, according to the recommendations of Quigley et al, ${ }^{52}$ investigators should select the appraisal tool based on the study design. Based on the results revealed from scope searching, this systematic review will primarily consist of cross-sectional studies. Therefore, an adapted version of NOS scale for cross-sectional studies will be used as has been done previously, ${ }^{567}$ since the main versions of this tool were traditionally developed to evaluate cohort and case-control studies. This will enable us to assess bias in the most relevant components, specific to cross-sectional studies rather than observational study designs in general.

Using the published version of the NOS scale, studies are rated in three main domains by using eight items overall: selection (four items), comparability (one item) and exposure (three items). ${ }^{45}$ One star can be given for the first and third categories, while up to two stars can be awarded for comparability. ${ }^{45}$ Therefore, the NOS scores range from 0 to 9 with the latter representing the highest quality. ${ }^{45}$ In the adapted version of the NOS scale for cross-sectional studies we will follow the same star ranking system as in the published version, however the 'exposure' domain of the original scale, will be modified to 'outcome. ${ }^{56}$ Modifications will be kept to minimum to ensure that the tool will remain close to the validated format. Depending on the number of stars that have been assigned to each study, then its quality will be converted to 'good', 'fair' and 'poor' in accordance with the Agency for Healthcare Research and Quality standards. ${ }^{39} 58$ The conversion thresholds used for this will be based on previous studies. ${ }^{59}$ Information regarding the scoring of each study and ROB will be collated and presented into a table in detail.

\section{Data synthesis}

The two reviewers will independently organise studies that are more homogeneous (clinically) in smaller groups according to the following factors:

- Type of pain (experimental or clinical, acute or chronic).

- The measurement of torque or force steadiness outcome (relative or absolute variability).

- The muscles and/or joints included in the measurement.

- Task characteristics (eg, sustained, dynamic, MVC target level).

- Feedback or no feedback.

This will take place, prior to the synthesis of the findings of the studies. The final decision of the subgrouping plan will be common between the two reviewers and any disagreement between the two will be resolved with discussion 
first and the third reviewer will mediate if needed. Pain will be regarded as chronic if it lasts longer than 3 months (ie, beyond normal healing time). ${ }^{60}$ Acute pain is 'the physiological response and experience to noxious stimuli that can become pathologic, is normally sudden in onset, time limited (commonly less than 1 month), and motivates behaviours to avoid actual or potential tissue injuries'. ${ }^{6162}$ Therefore, for the purposes of this systematic review, pain will be considered as acute if it lasts for less than 3 months.

When the included studies will be organised in the aforementioned subgroupings, the statistical heterogeneity of each subgroup will be quantified, using the $\mathrm{I}^{2}$ statistic. ${ }^{63}$ Values of $\mathrm{I}^{2}$ lower than $50 \%$ will be considered as eligible for meta-analysis. ${ }^{64}$ Then, a random-effects metaanalysis will be performed for each subgroup, according to specific guidelines ${ }^{33}{ }^{65}$ and the necessary data will be extracted (eg, estimate of effect, variance, etc). All data will then be transformed into a common rubric, which will most likely be odds ratios with $95 \%$ confidence intervals since we will most likely deal with binary data (ie, increase or decrease of force or torque steadiness) ${ }^{66}$ The robustness of the results will be assessed with a sensitivity analysis. ${ }^{60}$ This will be achieved by investigating whether ROB (excluding studies that were characterised as high ROB) and/or missing data (excluding studies in which mean and/or variance values were missing) have an impact on the meta-analysis. ${ }^{65} 67$

However, scoping searches of the current literature revealed that clinical diversity (eg, variability in terms of population, exposure, etc) and methodological diversity (eg, variability in terms of ROB) exists among the potentially eligible studies. Therefore, undertaking a meta-analysis may not be possible. In this case, a narrative synthesis approach will be used instead in this review and the synthesis process will follow specific guidelines. ${ }^{66} 68$ More specifically, the results will be described narratively in text or tabular form. A vote-counting synthesis method will be also used, based on the direction of effect and not on statistical significance or subjective rules which have been previously shown to be problematic. ${ }^{669}$ Appropriate methods will be used to display and present the data. It is most likely that this will include an effect direction plot, which will be used to present the results of the synthesis in a form as the following: 'pain increases force steadiness', 'no difference', 'pain decreases force steadiness'. ${ }^{70}$ Any changes or additional decisions made regarding the data organisation and presentation at review stage though, based on the actual review findings, will be reported and justified.

\section{Confidence in cumulative evidence}

To ensure the minimisation of ROB, the guidelines provided by Page et al (2019) ${ }^{72}$ in combination with the steps that have already been described in the information source section will be followed. Roughly, this will consist of a comprehensive search (including grey literature, protocol registers and other), attempts to obtain unpublished data by contacting investigators and identifying studies that have not been published yet by contacting notable authors of the field. ${ }^{72}$ If deemed necessary, funnel plots will be used to determine the extent of the risk. ${ }^{72}$

Finally, the Grading of Recommendations Assessment, Development and Evaluation approach will be used to appraise the evidence, in terms of cumulative strength and quality ${ }^{73} 74$ This will include five steps as described by Goldet and Howick ${ }^{75}$ and the final quality of evidence will be presented as 'high', 'moderate', 'low' or 'very low'. According to these guidelines, observational studies will be given an a priori rating of low quality and from that point their quality will be either upgraded or downgraded. ${ }^{75}$ Reasons to upgrade the certainty of evidence will include large effect sizes and dose-response relationships between pain and force steadiness, while ROB, inconsistencies between studies, imprecision, indirectness of evidence and publication bias will be reasons to downgrade the certainty of evidence. ${ }^{76}$ This classification of the cumulative quality of evidence will be completed for the combined grouping for all studies within the review and for each subgroup of studies, as described previously. Based on this assessment of the evidence, recommendations for the interpretation of the evidence will be given as described by Guyatt et al and Dekkers et $a l^{33} 77$

\section{Patient and public involvement}

This systematic review protocol was not developed following discussions within a patient and public involvement meeting (PPI), due to COVID-19. Patients will not be involved in the analysis and data collection of the systematic review. However, the results of this study will be shared at PPI meetings at the University of Birmingham in the future.

\section{Ethics and dissemination of results}

This systematic review will involve the collection of previously published studies, in which participants' decision for participation was informed and voluntary. Therefore, ethical approval is not required. Our findings will be presented at national and/or international conferences, while they will also be submitted for publication in a refereed journal.

Twitter Michail Arvanitidis @physio_arv, Deborah Falla @Deb_Falla, Andy Sanderson @AndyCSanderson and Eduardo Martinez-Valdes @mredumartinez

Contributors All authors have contributed to the design of this systematic review protocol. MA created the first draft of the protocol with guidance from all the other authors (ie, AS, DF and EM-V). All drafts were revised and reviewed by all authors before the final approval of the last version of the manuscript.

Funding The authors have not declared a specific grant for this research from any funding agency in the public, commercial or not-for-profit sectors.

Competing interests None declared.

Patient consent for publication Not required.

Provenance and peer review Not commissioned; externally peer reviewed.

Supplemental material This content has been supplied by the author(s). It has not been vetted by BMJ Publishing Group Limited (BMJ) and may not have been peer-reviewed. Any opinions or recommendations discussed are solely those 
of the author(s) and are not endorsed by BMJ. BMJ disclaims all liability and responsibility arising from any reliance placed on the content. Where the content includes any translated material, BMJ does not warrant the accuracy and reliability of the translations (including but not limited to local regulations, clinical guidelines, terminology, drug names and drug dosages), and is not responsible for any error and/or omissions arising from translation and adaptation or otherwise.

Open access This is an open access article distributed in accordance with the Creative Commons Attribution Non Commercial (CC BY-NC 4.0) license, which permits others to distribute, remix, adapt, build upon this work non-commercially, and license their derivative works on different terms, provided the original work is properly cited, appropriate credit is given, any changes made indicated, and the use is non-commercial. See: http://creativecommons.org/licenses/by-nc/4.0/.

\section{ORCID iDs}

Michail Arvanitidis http://orcid.org/0000-0002-3339-6668

Deborah Falla http://orcid.org/0000-0003-1689-6190

Andy Sanderson http://orcid.org/0000-0002-7892-1067

Eduardo Martinez-Valdes http://orcid.org/0000-0002-5790-7514

\section{REFERENCES}

1 Enoka RM, Christou EA, Hunter SK, et al. Mechanisms that contribute to differences in motor performance between young and old adults. J Electromyogr Kinesiol 2003;13:1-12.

2 Oomen NMCW, van Dieën JH. Effects of age on force steadiness: a literature review and meta-analysis. Ageing Res Rev 2017;35:312-21.

3 Tracy BL, Enoka RM. Older adults are less steady during submaximal isometric contractions with the knee extensor muscles. J Appl Physiol 2002;92:1004-12.

4 Tracy BL. Force control is impaired in the ankle plantarflexors of elderly adults. Eur J Appl Physiol 2007;101:629-36.

5 Maenhout AG, Palmans T, De Muynck M, et al. The impact of rotator cuff tendinopathy on proprioception, measuring force sensation. $J$ Shoulder Elbow Surg 2012;21:1080-6.

6 Pua Y-H, Ong P-H, Ho J-Y, et al. Associations of isokinetic knee steadiness with hop performance in patients with ACL deficiency. Knee Surg Sports Traumatol Arthrosc 2015;23:2185-95.

7 Deering RE, Senefeld JW, Pashibin T, et al. Muscle function and fatigability of trunk flexors in males and females. Biol Sex Differ 2017;8:12.

8 Testa M, Geri T, Pitance L, et al. Alterations in jaw clenching force control in people with myogenic temporomandibular disorders. $J$ Electromyogr Kinesiol 2018;43:111-7.

9 Ager AL, Borms D, Deschepper L. Proprioception: how is it affected by shoulder pain? A systematic review. J Hand Ther 2019; S08941130:30094-8.

10 Proske U, Gandevia SC. The proprioceptive senses: their roles in signaling body shape, body position and movement, and muscle force. Physiol Rev 2012:92:1651-97.

11 Röijezon U, Clark NC, Treleaven J. Proprioception in musculoskeletal rehabilitation. Part 1: basic science and principles of assessment and clinical interventions. Man Ther 2015;20:368-77.

12 Fortier S, Basset FA. The effects of exercise on limb proprioceptive signals. J Electromyogr Kinesiol 2012;22:795-802.

13 Madsen BK, Søgaard K, Andersen LL, et al. Neck/shoulder function in tension-type headache patients and the effect of strength training $J$ Pain Res 2018;11:445-54.

14 Martinez-Valdes E, Negro F, Falla D, et al. Inability to increase the neural drive to muscle is associated with task failure during submaximal contractions. J Neurophysiol 2020;124:1110-21.

15 Fiogbé E, Carnavale BF, Takahashi ACdeM. Exercise training in older adults, what effects on muscle force control? A systematic review of randomized clinical trials. Arch Gerontol Geriatr 2019;83:138-50.

16 Tracy BL. Visuomotor contribution to force variability in the plantarflexor and dorsiflexor muscles. Hum Mov Sci 2007;26:796-807.

17 Hodges PW, Tucker K. Moving differently in pain: a new theory to explain the adaptation to pain. Pain 2011;152:S90-8.

18 Tucker K, Larsson A-K, Oknelid S, et al. Similar alteration of motor unit recruitment strategies during the anticipation and experience of pain. Pain 2012;153:636-43.

19 Sjölander P, Michaelson P, Jaric S, et al. Sensorimotor disturbances in chronic neck pain--range of motion, peak velocity, smoothness of movement, and repositioning acuity. Man Ther 2008;13:122-31.

20 Treleaven J. Sensorimotor disturbances in neck disorders affecting postural stability, head and eye movement control. Man Ther 2008;13:2-11.
21 Lee AS, Cholewicki J, Reeves NP, et al. Comparison of trunk proprioception between patients with low back pain and healthy controls. Arch Phys Med Rehabil 2010;91:1327-31.

22 Salahzadeh Z, Maroufi N, Salavati M, et al. Proprioception in subjects with Patellofemoral pain syndrome: using the sense of force accuracy. J Musculoskelet Pain 2013;21:341-9.

23 Muceli S, Farina D, Kirkesola G, et al. Reduced force steadiness in women with neck pain and the effect of short term vibration. $J$ Electromyogr Kinesiol 2011;21:283-90.

24 Ferreira AS, de Oliveira Silva D, Ferrari D, et al. Knee and hip isometric force Steadiness are impaired in women with Patellofemoral pain. J Strength Cond Res 2019. doi:10.1519/ JSC.0000000000003215. [Epub ahead of print: 22 Jul 2019].

25 Del Santo F, Gelli F, Spidalieri R, et al. Corticospinal drive during painful voluntary contractions at constant force output. Brain Res 2007;1128:91-8.

26 Bandholm T, Rasmussen L, Aagaard P, et al. Effects of experimental muscle pain on shoulder-abduction force steadiness and muscle activity in healthy subjects. Eur J Appl Physiol 2008;102:643-50.

27 Salomoni SE, Graven-Nielsen T. Experimental muscle pain increases normalized variability of multidirectional forces during isometric contractions. Eur J Appl Physiol 2012;112:3607-17.

28 Martinez-Valdes E, Negro F, Farina D, et al. Divergent response of low- versus high-threshold motor units to experimental muscle pain. J Physiol 2020;598:2093-108.

29 Mista CA, Monterde S, Inglés M, et al. Reorganized force control in elbow pain patients during isometric wrist extension. Clin $\mathrm{J}$ Pain 2018;34:732-8.

30 Zanca GG, Saccol MF, Oliveira AB, et al. Shoulder internal and external rotations torque steadiness in overhead athletes with and without impingement symptoms. J Sci Med Sport 2013;16:433-7.

31 Bandholm T, Rasmussen L, Aagaard P, et al. Force steadiness, muscle activity, and maximal muscle strength in subjects with subacromial impingement syndrome. Muscle Nerve 2006;34:631-9.

32 Hortobágyi T, Garry J, Holbert D, et al. Aberrations in the control of quadriceps muscle force in patients with knee osteoarthritis. Arthritis Rheum 2004;51:562-9.

33 Dekkers OM, Vandenbroucke JP, Cevallos M, et al. COSMOS-E: guidance on conducting systematic reviews and meta-analyses of observational studies of etiology. PLoS Med 2019;16:e1002742.

34 Moher D, Shamseer L, Clarke M, et al. Preferred reporting items for systematic review and meta-analysis protocols (PRISMA-P) 2015 statement. Syst Rev 2015;4:1.

35 Shamseer L, Moher D, Clarke M, et al. Preferred reporting items for systematic review and meta-analysis protocols (PRISMA-P) 2015: elaboration and explanation. BMJ 2015;350:g7647.

36 Higgins JPT, Thomas J, Chandler J. Cochrane Handbook for systematic reviews of interventions version 6.0 (updated July 2019). Cochrane, 2019. Available: www.training.cochrane.org/ handbook

37 Mueller M, D'Addario M, Egger M, et al. Methods to systematically review and meta-analyse observational studies: a systematic scoping review of recommendations. BMC Med Res Methodol 2018;18:44.

38 Smith V, Devane D, Begley CM, et al. Methodology in conducting a systematic review of systematic reviews of healthcare interventions. BMC Med Res Methodol 2011;11:15.

39 Devecchi V, Gallina A, Heneghan NR, et al. Are neuromuscular adaptations present in people with recurrent spinal pain during a period of remission? A protocol for a systematic review. BMJ Open 2019;9:e033276.

40 Richter RR, Austin TM. Using mesh (medical subject headings) to enhance PubMed search strategies for evidence-based practice in physical therapy. Phys Ther 2012;92:124-32.

41 et alLefebvre C, Glanville J, Briscoe S. Chapter 4: searching for and selecting studies. in: Higgins JPT, Thomas J, Chandler J, et al. (editors). Cochrane Handbook for systematic reviews of interventions version 6.0 (updated July 2019). Cochrane, 2019. Available: www. training.cochrane.org/handbook

42 Jenuwine ES, Floyd JA. Comparison of medical subject Headings and text-word searches in MEDLINE to retrieve studies on sleep in healthy individuals. J Med Libr Assoc 2004;92:349-53.

43 Li T, Higgins JPT, Deeks JJ. (editors). Chapter 5: collecting data. in: Higgins JPT, Thomas J, Chandler J, et al. (editors). Cochrane Handbook for systematic reviews of interventions version 6.0 (updated July 2019). Cochrane, 2019. Available: www.training. cochrane.org/handbook

44 Orwin R. Evaluating coding decisions. In: Cooper H, Hedges L, eds. The Handbook of research synthesis. New York: Russell Sage Foundation, 1994.

45 Wells GA, Shea B, O'Connell D. The Newcastle-Ottawa scale (NOS) for assessing the quality of nonrandomised studies in meta- 
analyses. available. Available: http://www.ohri.ca/programs/clinical_ epidemiology/oxford.asp [Accessed 05 May 2020].

46 Sanderson S, Tatt ID, Higgins JPT. Tools for assessing quality and susceptibility to bias in observational studies in epidemiology: a systematic review and annotated bibliography. Int J Epidemiol 2007;36:666-76.

47 Losilla J-M, Oliveras I, Marin-Garcia JA, et al. Three risk of bias tools lead to opposite conclusions in observational research synthesis. $J$ Clin Epidemiol 2018;101:61-72.

48 Lang S, Kleijnen J. Quality assessment tools for observational studies: lack of consensus. Int J Evid Based Healthc 2010;8:247.

49 Seehra J, Pandis N, Koletsi D, et al. Use of quality assessment tools in systematic reviews was varied and inconsistent. J Clin Epidemiol 2016;69:179-84.

50 Waddington $\mathrm{H}$, Aloe AM, Becker BJ, et al. Quasi-Experimental study designs series-paper 6: risk of bias assessment. J Clin Epidemiol 2017;89:43-52.

51 Briere J-B, Bowrin K, Taieb V, et al. Meta-Analyses using real-world data to generate clinical and epidemiological evidence: a systematic literature review of existing recommendations. Curr Med Res Opin 2018;34:2125-30.

52 Quigley JM, Thompson JC, Halfpenny NJ, et al. Critical appraisal of nonrandomized studies-A review of recommended and commonly used tools. J Eval Clin Pract 2019;25:44-52.

53 Hootman JM, Driban JB, Sitler MR, et al. Reliability and validity of three quality rating instruments for systematic reviews of observational studies. Res Synth Methods 2011;2:110-8.

54 Hartling L, Milne A, Hamm MP, et al. Testing the Newcastle Ottawa scale showed low reliability between individual reviewers. J Clin Epidemiol 2013;66:982-93.

55 Farrah K, Young K, Tunis MC, et al. Risk of bias tools in systematic reviews of health interventions: an analysis of PROSPERO-registered protocols. Syst Rev 2019;8:280.

56 Herzog R, Álvarez-Pasquin MJ, Díaz C, et al. Are healthcare workers' intentions to vaccinate related to their knowledge, beliefs and attitudes? A systematic review. BMC Public Health 2013;13:154.

57 Rostami M, Younesi SJ, Mohammadi Shahboulaghi F, et al. Models of suicide in elderly: a protocol for a systematic review. BMJ Open 2018;8:e022087.

58 Sanderson A, Rushton AB, Martinez Valdes E, et al. The effect of chronic, non-specific low back pain on superficial lumbar muscle activity: a protocol for a systematic review and meta-analysis. BMJ Open 2019;9:e029850.

59 Eckard TG, Padua DA, Hearn DW, et al. The relationship between training load and injury in athletes: a systematic review. Sports Med 2018;48:1929-61.

60 Treede R-D, Rief W, Barke A, et al. A classification of chronic pain for ICD-11. Pain 2015;156:1-7.

61 Carr DB, Goudas LC. Acute pain. Lancet 1999;353:2051-8.

62 Tighe P, Buckenmaier CC, Boezaart AP, et al. Acute pain medicine in the United States: a status report. Pain Med 2015;16:1806-26.

63 Higgins JPT, Thompson SG, Deeks JJ, et al. Measuring inconsistency in meta-analyses. BMJ 2003;327:557-60.

64 Heneghan NR, Smith R, Rushton A. Thoracic dysfunction in whiplash-associated disorders: a systematic review and metaanalysis protocol. Syst Rev 2016;5:26.

65 Deeks JJ, Higgins JPT, Altman DG. Chapter 10: analysing data and undertaking meta-analyses. in: Higgins JPT, Thomas J, Chandler $\mathrm{J}$, et al. (editors). Cochrane Handbook for systematic reviews of interventions version 6.0 (updated July 2019). Cochrane, 2019. available from. Available: www.training.cochrane.org/handbook

66 Popay J, Roberts H, Sowden A. Guidance on the conduct of narrative synthesis in systematic reviews: a product from the ESRC methods programme. Lancaster, UK: Lancaster University, 2006.

67 Price J, Rushton A, Tyros I, et al. Effectiveness and optimal dosage of resistance training for chronic non-specific neck pain: a protocol for a systematic review with a qualitative synthesis and meta-analysis. BMJ Open 2019;9:e025158.

68 et alMcKenzie JE, Brennan SE, Ryan RE. Chapter 9: Summarizing study characteristics and preparing for synthesis. in: Higgins JPT, Thomas J, Chandler J, et al. (editors). Cochrane Handbook for systematic reviews of interventions version 6.0 (updated July 2019). Cochrane, 2019. Available: www.training.cochrane.org/ handbook

69 loannidis JPA, Patsopoulos NA, Rothstein HR. Reasons or excuses for avoiding meta-analysis in forest plots. BMJ 2008;336:1413-5.

70 Thomson HJ, Thomas S. The effect direction plot: visual display of non-standardised effects across multiple outcome domains. Res. Syn. Meth. 2013;4:95-101.

71 McKenzie JE, Brennan SE. Chapter 12: synthesizing and presenting findings using other methods. in: Higgins JPT, Thomas J, Chandler $\mathrm{J}$, et al. (editors). Cochrane Handbook for systematic reviews of interventions version 6.0 (updated July 2019). Cochrane, 2019. Available: www.training.cochrane.org/handbook

72 Page MJ, Higgins JPT, Sterne JAC. Chapter 13: assessing risk of bias due to missing results in a synthesis. in: Higgins JPT, Thomas $\mathrm{J}$, Chandler J, et al. (editors). Cochrane Handbook for systematic reviews of interventions version 6.0 (updated July 2019). Cochrane, 2019. Available: www.training.cochrane.org/handbook

73 Guyatt GH, Oxman AD, Vist G, et al. Grade guidelines: 4. rating the quality of evidence-study limitations (risk of bias). J Clin Epidemiol $2011 ; 64: 407-15$.

74 Schünemann H, Brożek J, Guyatt G. Eds. Handbook for grading the quality of evidence and the strength of recommendations using the grade approach, 2013. Available: https://gdt.gradepro.org/app/ handbook/ handbook.html

75 Goldet G, Howick J. Understanding grade: an introduction. J Evid Based Med 2013;6:50-4.

76 Balshem H, Helfand M, Schünemann HJ, et al. Grade guidelines: 3. rating the quality of evidence. J Clin Epidemiol 2011;64:401-6.

77 Guyatt GH, Oxman AD, Vist GE, et al. Grade: an emerging consensus on rating quality of evidence and strength of recommendations. BMJ 2008;336:924-6.

78 Perrot S, Cohen M, Barke A. IASP Taskforce for the classification of chronic pain. The IASP classification of chronic pain for ICD-11: chronic secondary musculoskeletal pain. Pain 2019;160:77-82.

79 Nicholas M, Vlaeyen JWS, Rief W. IASP Taskforce for the classification of chronic pain. The IASP classification of chronic pain for ICD-11: chronic primary pain. Pain 2019;160:28-37.

80 Reddy KSK, Naidu MUR, Rani PU, et al. Human experimental pain models: a review of standardized methods in drug development. $J$ Res Med Sci 2012;17:587-95.

81 Rice DA, McNair PJ, Lewis GN, et al. Experimental knee pain impairs submaximal force steadiness in isometric, eccentric, and concentric muscle actions. Arthritis Res Ther 2015;17:259.

82 Li K, Evans PJ, Seitz WH, et al. Carpal tunnel syndrome impairs sustained precision pinch performance. Clin Neurophysiol 2015;126:194-201. 